\title{
Hidden-Beauty Charged Tetraquarks and Heavy Quark Spin Conservation
}

\author{
A. Ali ${ }^{\Uparrow}$, L. Maiani*, A.D. Polosa*, V. Riquer* \\ "Deutsches Elektronen-Synchrotron DESY, D-22607 Hamburg, Germany \\ * Dipartimento di Fisica, Sapienza Università di Roma, Piazzale Aldo Moro 2, I-00185 Roma, Italy \\ and INFN Sezione di Roma, Piazzale Aldo Moro 2, I-00185 Roma, Italy
}

\begin{abstract}
Assuming the dominance of the spin-spin interaction in a diquark, we point out that the mass difference in the beauty sector $M\left(Z_{b}^{\prime}\right)^{ \pm}-M\left(Z_{b}\right)^{ \pm}$scales with quark masses as expected in QCD, with respect to the corresponding mass difference $M\left(Z_{c}^{\prime}\right)^{ \pm}-M\left(Z_{c}\right)^{ \pm}$. Notably, we show that the decays $\Upsilon(10890) \rightarrow \Upsilon(n S) \pi^{+} \pi^{-}$and $\Upsilon(10890) \rightarrow\left(h_{b}(1 P), h_{b}(2 P)\right) \pi^{+} \pi^{-}$are compatible with heavy-quark spin conservation if the contributions of $Z_{b}, Z_{b}^{\prime}$ intermediate states are taken into account, $\Upsilon(10890)$ being either a $\Upsilon(5 S)$ or the beauty analog of $Y_{c}(4260)$. Belle results on these decays support the quark spin wave-function of the $\mathrm{Z}$ states as tetraquarks. We also consider the role of light quark spin non-conservaton in $Z_{b}, Z_{b}^{\prime}$ decays into $B B^{*}$ and $B^{*} B^{*}$. Indications of possible signatures of the still missing $X_{b}$ resonance are proposed.
\end{abstract}

Preprint \# DESY 14-234 
Tetraquark interpretation of the hidden charm and beauty exotic resonances has been advanced and studied in considerable detail (see Refs. [1] 2], and [3]). In a recent contribution [4, a new scheme for the spin-spin quark interactions in the hidden charm resonances has been proposed, which reproduces well the mass and decay pattern of $X(3872)$, of the recently discovered [5] $Z_{c}^{ \pm, 0}(3900), Z_{c}^{ \pm, 0}(4020)$, and of the lowest lying $J^{P C}=1^{--} Y$ states.

Tetraquark states in the large $N_{c}$ (color) limit of QCD have been considered in [6] and [7] (see also the review [8] and references therein). Compact tetraquark mesons may have decay widths as narrow as $1 / N_{c}$, contrary to previous beliefs, and therefore they are reasonable candidates for a secondary spectroscopic meson series, in addition to the standard $q \bar{q}$ one. Another route to multiquark meson states is being explored in [9] within the Born-Oppenheimer approximation of QCD , where examples of selection rules for hadronic transitions have been worked out.

In this letter we consider the extension of the scheme presented in [4 for the hidden-charm to the hidden-beauty resonances $Z_{b}^{ \pm, 0}(10610)=Z_{b}$ and $Z_{b}^{ \pm, 0}(10650)=Z_{b}^{\prime}$.

These resonances are interpreted as $S$-wave $J^{P G}=1^{++}$states with diquark spin distribution (use the notation $\left|s_{[b q]}, s_{[\bar{b} \bar{q}]}\right\rangle$ for diquark spins)

$$
\begin{aligned}
\left|Z_{b}\right\rangle & =\frac{\left|1_{b q}, 0_{\bar{b} \bar{q}}\right\rangle-\left|0_{b q}, 1_{\bar{b} \bar{q}}\right\rangle}{\sqrt{2}} \\
\left|Z_{b}^{\prime}\right\rangle & =\left|1_{b q}, 1_{\bar{b} \bar{q}}\right\rangle_{J=1}
\end{aligned}
$$

The $J^{P}=1^{+}$multiplet is completed by $X_{b}$, which is given by the $C=+1$ combination

$$
\left|X_{b}\right\rangle=\frac{\left|1_{b q}, 0_{\bar{b} \bar{q}}\right\rangle+\left|0_{b q}, 1_{\bar{b} \bar{q}}\right\rangle}{\sqrt{2}}
$$

Assuming the spin-spin interaction inside diquarks to dominate, we expect $X_{b}$ and $Z_{b}$ to be degenerate, with $Z_{b}^{\prime}$ heavier according to

$$
M\left(Z_{b}^{\prime}\right)-M\left(Z_{b}\right)=2 \kappa_{b}
$$

where $\kappa_{b}$ is the strength of the spin-spin interaction inside the diquark. A similar analysis for the hidden-charm resonances has produced the value [4]

$$
2 \kappa_{c}=M\left(Z_{c}^{\prime}\right)-M\left(Z_{c}\right) \simeq 120 \mathrm{MeV}
$$

The QCD expectation is $\kappa_{b}: \kappa_{c}=M_{c}: M_{b}$. The ratio can be estimated from the masses reported in [10]

$$
\frac{M_{c}}{M_{b}} \simeq \frac{1.27}{4.18}=0.30
$$

giving $2 \kappa_{b} \simeq 36 \mathrm{MeV}$, which fits nicely with the observed $Z_{b}^{\prime}-Z_{b}$ mass difference $(\simeq 45 \mathrm{MeV})$.

Next we consider another crucial prediction of QCD, namely conservation of the heavy quark spin in hadronic decays. 
We recall that $Z_{b}, Z_{b}^{\prime}$ are observed in the decays of $\Upsilon(10890)$

$$
\Upsilon(10890) \rightarrow Z_{b} / Z_{b}^{\prime}+\pi \rightarrow h_{b}(n P) \pi \pi
$$

The $\Upsilon(10890)$ is usually reported as the $\Upsilon(5 S)$ since its mass is close to the mass of the $5 S$ state predicted by potential models. However, a different assignment was proposed in [11], namely $\Upsilon(10890)=Y_{b}$, the latter state being a $P$-wave tetraquark analogous to the $Y(4260)$. A reason for this assignment is the analogy of $\Upsilon(10890)$ decay (6) with $Y(4260) \rightarrow Z_{c}(3900)+\pi$, with $Y(4260)$ being the the first discovered $Y$ state [12]. Current experimental situation about $\Upsilon(10890)$ is still in a state of flux. In our opinion, the possibility that $\Upsilon(10890)$ is an unresolved peak involving both the $\Upsilon(5 S)$ and $Y_{b}$, reported by Belle some time ago [13], is plausible, providing a resolution of the observed branching ratios measured at the $\Upsilon(10890)$ [14. However, this identification is not a requirement in the considerations presented below. In fact, following the assignment of $Y(4260)$ as a $P$-wave tetraquark with $s_{c \bar{c}}=1$ [4], one sees that in both cases the initial state in (6) corresponds to $s_{b \bar{b}}=1$. As is well known $h_{b}(n P)$ has $s_{b \bar{b}}=0$, pointing to a possible violation of the heavy-quark spin conservation, as suggested in [14].

We show now that the contradiction is only apparent. Expressing the states in the the basis of definite $b \bar{b}$ and $q \bar{q}$ spin, one finds

$$
\begin{aligned}
\left|Z_{b}\right\rangle & =\frac{\left|1_{q \bar{q}}, 0_{b \bar{b}}\right\rangle-\left|0_{q \bar{q}}, 1_{b \bar{b}}\right\rangle}{\sqrt{2}} \\
\left|Z_{b}^{\prime}\right\rangle & =\frac{\left|1_{q \bar{q}}, 0_{b \bar{b}}\right\rangle+\left|0_{q \bar{q}}, 1_{b \bar{b}}\right\rangle}{\sqrt{2}}
\end{aligned}
$$

It is conceivable that the subdominant spin-spin interactions may play a non negligible role in the $b$-systems, as the spin-spin dominant interaction is suppressed by the large $b$ quark mass. In this case the composition of $Z_{b}, Z_{b}^{\prime}$ indicated in Eq. (7) would be more general:

$$
\begin{aligned}
\left|Z_{b}\right\rangle & =\frac{\alpha\left|1_{q \bar{q}}, 0_{b \bar{b}}\right\rangle-\beta\left|0_{q \bar{q}}, 1_{b \bar{b}}\right\rangle}{\sqrt{2}} \\
\left|Z_{b}^{\prime}\right\rangle & =\frac{\beta\left|1_{q \bar{q}}, 0_{b \bar{b}}\right\rangle+\alpha\left|0_{q \bar{q}}, 1_{b \bar{b}}\right\rangle}{\sqrt{2}}
\end{aligned}
$$

The kets in (7) and (8) represent a superposition of color singlet and color octet. These states evolve, by QCD interactions, into pairs of color singlet mesons with heavy quark spin conserved in the limit $M_{b} \rightarrow \infty$, e.g. $\left|1_{q \bar{q}}, 0_{b \bar{b}}\right\rangle$ gives rise to $\eta_{b}, h_{b}$ etc., but not $\Upsilon_{b}, \chi_{j b}$ etc.. No similar constraint applies to the light quark spin.

We assume $\alpha$ and $\beta$ to be both different from zero. If either one of the two vanishes, the decay (6) would be altogether forbidden by heavy quark spin conservation, contrary to what is observed for the distribution of $M\left(h_{b} \pi\right)$ in Ref. [15].

Define

$$
\begin{aligned}
& g_{Z}=g\left(\Upsilon \rightarrow Z_{b} \pi\right) g\left(Z_{b} \rightarrow h_{b} \pi\right) \propto-\alpha \beta\left\langle h_{b} \mid 1_{q \bar{q}}, 0_{b \bar{b}}\right\rangle\left\langle 0_{q \bar{q}}, 1_{b \bar{b}} \mid \Upsilon\right\rangle \\
& g_{Z^{\prime}}=g\left(\Upsilon \rightarrow Z_{b}^{\prime} \pi\right) g\left(Z_{b}^{\prime} \rightarrow h_{b} \pi\right) \propto \alpha \beta\left\langle h_{b} \mid 1_{q \bar{q}}, 0_{b \bar{b}}\right\rangle\left\langle 0_{q \bar{q}}, 1_{b \bar{b}} \mid \Upsilon\right\rangle
\end{aligned}
$$




\begin{tabular}{|c|c|c|c|c|c|}
\hline Final State & $\Upsilon(1 S) \pi^{+} \pi^{-}$ & $\Upsilon(2 S) \pi^{+} \pi^{-}$ & $\Upsilon(3 S) \pi^{+} \pi^{-}$ & $h_{b}(1 P) \pi^{+} \pi^{-}$ & $h_{b}(2 P) \pi^{+} \pi^{-}$ \\
\hline \hline Rel. Norm. & $0.57 \pm 0.21_{-0.04}^{+0.19}$ & $0.86 \pm 0.11_{-0.10}^{+0.04}$ & $0.96 \pm 0.14_{-0.05}^{+0.08}$ & $1.39 \pm 0.37_{-0.15}^{+0.05}$ & $1.6_{-0.4-0.6}^{+0.6+0.4}$ \\
\hline Rel. Phase & $58 \pm 43_{-9}^{+4}$ & $-13 \pm 13_{-8}^{+17}$ & $-9 \pm 19_{-26}^{+11}$ & $187_{-57-12}^{+44+3}$ & $181_{-105-109}^{+665+74}$ \\
\hline
\end{tabular}

Table 1: Values of the relative normalizations and of the relative phases (in degrees), for $s_{b \bar{b}}: 1 \rightarrow 1$ and $1 \rightarrow 0$ transitions, as reported by $[15]$.

where $g$ are the effective strong couplings at the vertices $\Upsilon Z_{b} \pi$ and $Z_{b} h_{b} \pi$. For both assignments of $\Upsilon(10890)$, Eq. (8) and heavy quark spin conservation require

$$
g_{Z}=-g_{Z^{\prime}}
$$

independently from the values of the mixing coefficients $\alpha, \beta$.

In Ref. [15] the amplitude for the decay (6) is fitted with two Breit-Wigners corresponding to the $Z_{b}, Z_{b}^{\prime}$ intermediate states. Table I therein, that we transcribe here in Table 1 , shows the relative normalizations and phases obtained by the fit, for decays into $h_{b}(1 P)$ and $h_{b}(2 P)$. Within large errors, consistency with Eq. (99), that is with the heavy-quark spin conservation, is apparent.

It is interesting that the same conclusion was drawn using a picture in which $Z_{b}, Z_{b^{\prime}}$ have a "molecular" type structure [16]

$$
Z_{b}=\frac{\left|B, \bar{B}^{*}\right\rangle-\left|\bar{B}, B^{*}\right\rangle}{\sqrt{2}} \quad Z_{b}^{\prime}=\left|B^{*}, \bar{B}^{*}\right\rangle_{J=1}
$$

To determine $\alpha$ and $\beta$ separately, one has to resort to $s_{b \bar{b}}: 1 \rightarrow 1$ transitions, such as

$$
\Upsilon(10890) \rightarrow Z_{b} / Z_{b}^{\prime}+\pi \rightarrow \Upsilon(n S) \pi \pi(n=1,2,3)
$$

The effective couplings analogous to (9) are

$$
\begin{aligned}
& f_{Z}=f\left(\Upsilon \rightarrow Z_{b} \pi\right) f\left(Z_{b} \rightarrow \Upsilon(n S) \pi\right) \propto|\beta|^{2}\left\langle\Upsilon(n S) \mid 0_{q \bar{q}}, 1_{b \bar{b}}\right\rangle\left\langle 0_{q \bar{q}}, 1_{b \bar{b}} \mid \Upsilon\right\rangle \\
& f_{Z^{\prime}}=f\left(\Upsilon \rightarrow Z_{b}^{\prime} \pi\right) f\left(Z_{b}^{\prime} \rightarrow \Upsilon(n S) \pi\right) \propto|\alpha|^{2}\left\langle\Upsilon(n S) \mid 0_{q \bar{q}}, 1_{b \bar{b}}\right\rangle\left\langle 0_{q \bar{q}}, 1_{b \bar{b}} \mid \Upsilon\right\rangle
\end{aligned}
$$

The Dalitz plot of these decays indicate indeed that the transitions (12) proceed mainly through $Z_{b}$ and $Z_{b}^{\prime}$ [14,15], though the amplitude for the process $\Upsilon(10890) \rightarrow \Upsilon(1 S) \pi^{+} \pi^{-}$has a significant direct component, which is expected in the tetraquark interpretation of the state $\Upsilon(10890)[3$. This is also reflected in Table 1. A quantitative analysis of the Belle data including the direct and resonant components (i.e., via the intermediate resonant states $Z_{b}$ and $Z_{b}^{\prime}$ ) is required to test the 
underlying dynamics. Leaving this for the future, we argue here that parametrizing the amplitude in terms of two Breit-Wigner as before, one determines the ratio $\alpha / \beta$. Indeed, from the Belle results [15] we find the following weighted average values: 1:

$$
\begin{aligned}
& s_{b \bar{b}}: 1 \rightarrow 1 \text { transition : } \\
& \overline{\text { Rel.Norm. }}=0.85 \pm 0.08=|\alpha|^{2} /|\beta|^{2} \\
& \overline{\text { Rel.Phase }}=(-8 \pm 10)^{\circ}
\end{aligned}
$$

and

$$
\begin{aligned}
& s_{b \bar{b}}: 1 \rightarrow 0 \text { transition : } \\
& \overline{\text { Rel.Norm. }}=1.4 \pm 0.3 \\
& \overline{\text { Rel.Phase }}=(185 \pm 42)^{\circ} .
\end{aligned}
$$

Within errors, the tetraquark assignment in Eqs. (11) and (7) with $\alpha=\beta=1$ is supported.

As a side remark, we observe that a Fierz rearrangement similar to the one used in (7) puts together $b \bar{q}$ and $q \bar{b}$ fields

$$
\begin{aligned}
\left|Z_{b}\right\rangle & =\left|1_{b \bar{q}}, 1_{q \bar{b}}\right\rangle_{J=1} \\
\left|Z_{b}^{\prime}\right\rangle & =\frac{\left|1_{b \bar{q}}, 0_{q \bar{b}}\right\rangle+\left|0_{b \bar{q}}, 1_{q \bar{b}}\right\rangle}{\sqrt{2}}
\end{aligned}
$$

The labels $0_{b \bar{q}}$ and $1_{b \bar{q}}$ could be viewed as indicating $B$ and $B^{*}$ mesons, respectively, leading to the prediction of the decay patterns $Z_{b} \rightarrow B^{*} \bar{B}^{*}$ and $Z_{b}^{\prime} \rightarrow B \bar{B}^{*}$ [3]. This would not be in agreement with the Belle data [14.

This argument, however, rests on conservation of the light quark spin which, unlike the heavy quark spin, may change when the color octet pairs which appear in (15), evolve into pairs of color singlet mesons. Therefore predictions derived from (15) are not as reliable as those derived from (7). We also stress that the issue of the unaccounted direct production of the $B^{*} \bar{B}^{*}, B \bar{B}^{*}$ and related states in the Belle data, collected at and near the $\Upsilon(10890)$ resonance [14, once satisfactorily resolved, may also reflect on the resonant contributions $Z_{b} \rightarrow B^{*} \bar{B}^{*}$ and $Z_{b}^{\prime} \rightarrow B \bar{B}^{*}$. We look forward to an improved analysis of the Belle measurements.

Finally we comment on the expected positive charge conjugation state, $X_{b}$. On the basis of the assumed spin-spin interaction, one predicts $M\left(X_{b}\right) \simeq M\left(Z_{b}\right) \simeq 10600 \mathrm{MeV}$. Such a state has been searched by CMS [17] in the region $10.1<M<11.0 \mathrm{GeV}$ and by ATLAS [18] in the region $10.5<M<11.0 \mathrm{GeV}$ looking for the decay

$$
X_{b} \rightarrow \Upsilon(1 S) \pi \pi
$$

so far with negative results.

In Ref. [2, it is noted that the near equality of the branching ratios for $X(3872) \rightarrow J / \psi 2 \pi$ and $X(3872) \rightarrow J / \psi 3 \pi$ can be understood if $X(3872)$ is predominantly isosinglet. The isospin

\footnotetext{
${ }^{1}$ for simplicity we have computed the weighted averages with statistical errors only.
} 
allowed decay in $J / \psi \omega$ is phase space forbidden and the decay in the $J / \psi \rho$ mode, although isospin forbidden, is phase space favoured, leading to similar rates.

In the $X_{b}$ decay, both $\omega$ and $\rho$ channels are allowed by phase space, so that, if $X_{b}$ is isosinglet, the dominant mode would be into $\Upsilon(1 S) \omega$. The suggestion therefore is to look at the decay $X_{b}(10600) \rightarrow \Upsilon(1 S) 3 \pi$ with the $3 \pi$ in the $\omega$ mass band, in parallel with the search for the $X_{b}(10600) \rightarrow \Upsilon(1 S) 2 \pi$ channel with the $2 \pi$ in the $\rho$ band. A search for $Y(10890) \rightarrow \gamma X_{b} \rightarrow$ $\gamma \omega \Upsilon(1 S)$ has been presented in Ref. [19] leading for the moment to an upper bound only.

\section{Acknowledgements}

We thank C. Hanhart for interesting discussions.

\section{References}

[1] L. Maiani, F. Piccinini, A. D. Polosa and V. Riquer, Phys. Rev. D 71, 014028 (2005) hep-ph/0412098.

[2] S. J. Brodsky, D. S. Hwang and R. F. Lebed, Phys. Rev. Lett. 113, 112001 (2014) arXiv:1406.7281 [hep-ph]].

[3] A. Ali, C. Hambrock, I. Ahmed and M. J. Aslam, Phys. Lett. B 684, 28 (2010) arXiv:0911.2787 [hep-ph]]; A. Ali, C. Hambrock and M. J. Aslam, Phys. Rev. Lett. 104, 162001 (2010) [Erratum-ibid. 107, 049903 (2011)] [arXiv:0912.5016 [hep-ph]].

[4] L. Maiani, F. Piccinini, A. D. Polosa and V. Riquer, Phys. Rev. D 89, 114010 (2014) arXiv:1405.1551 [hep-ph]].

[5] M. Ablikim et al. [BESIII Collaboration], Phys. Rev. Lett. 111, no. 24, 242001 (2013) arXiv:1309.1896 [hep-ex]]; M. Ablikim et al. [BESIII Collaboration], Phys. Rev. Lett. 113, no. 21, 212002 (2014) arXiv:1409.6577 [hep-ex]].

[6] S. Weinberg, Phys. Rev. Lett. 110, no. 26, 261601 (2013) [arXiv:1303.0342 [hep-ph]].

[7] M. Knecht and S. Peris, Phys. Rev. D 88, 036016 (2013) arXiv:1307.1273 [hep-ph]].

[8] A. Esposito, A. L. Guerrieri, F. Piccinini, A. Pilloni and A. D. Polosa, arXiv:1411.5997 [hepph]. See also N. Drenska, R. Faccini, F. Piccinini, A. Polosa, F. Renga and C. Sabelli, Riv. Nuovo Cim. 33, 633 (2010) arXiv:1006.2741 [hep-ph]].

[9] E. Braaten, C. Langmack and D. H. Smith, Phys. Rev. Lett. 112 (2014) 222001 arXiv:1401.7351 [hep-ph]].

[10] K. A. Olive et al. [Particle Data Group Collaboration], Chin. Phys. C 38, 090001 (2014). 
[11] A. Ali, C. Hambrock and W. Wang, Phys. Rev. D 85, 054011 (2012) [arXiv:1110.1333 [hep-ph]].

[12] B. Aubert et al. [BaBar Collaboration], Phys. Rev. Lett. 95, 142001 (2005) hep-ex/0506081. 13

[13] K.-F. Chen et al. [Belle Collaboration], Phys. Rev. D 82, 091106 (2010) arXiv:0810.3829 [hep-ex]].

[14] See talk of U. Tamponi at the 2014 Quarkonium Working Group held at CERN, 10-14 November 2014, https://indico.cern.ch/event/278195/other-view?view=standard

[15] A. Bondar et al. [Belle Collaboration], Phys. Rev. Lett. 108, 122001 (2012) arXiv:1110.2251 [hep-ex]].

[16] A. E. Bondar, A. Garmash, A. I. Milstein, R. Mizuk and M. B. Voloshin, Phys. Rev. D 84 (2011) 054010 arXiv:1105.4473 [hep-ph]].

[17] S. Chatrchyan et al. [CMS Collaboration], Phys. Lett. B 727, 57 (2013) arXiv:1309.0250 [hep-ex]].

[18] G. Aad et al. [ATLAS Collaboration], arXiv:1410.4409 [hep-ex].

[19] X. H. He et al. [Belle Collaboration], Phys. Rev. Lett. 113, no. 14, 142001 (2014) arXiv:1408.0504 [hep-ex]]. 